Sección uno: Ensayo

Educación y Exclusión social

\title{
Menores migrantes: Necesidades educativas y laborales detectadas a través de un estudio cualitativo ${ }^{1}$
}

Migrant minors: educational and labor needs detected through a qualitative study.

\author{
Jenifer Ortega Torres \\ Instituto de Migraciones Universidad \\ de Granada \\ jenniferortegatorres@gmail.com
}

\section{Resumen}

A lo largo de este artículo se llevará a cabo una aproximación a la realidad de jóvenes extutelados que, en su día, fueron acogidos en el Sistema de Protección de la Comunidad Autónoma de Andalucía en calidad de menores migrantes.

Esta aproximación se realizará por medio del análisis de 42 entrevistas realizadas a jóvenes extutelados, entre las que se encuentran 38 sujetos pertenecientes al sexo masculino y 4 al femenino, deteniéndonos en aquellos aspectos relacionados con lo educativo, prácticas formativas y lo laboral.

Las entrevistas a estos jóvenes se han realizado a través de las diferentes oleadas de trabajo de campo llevadas a cabo durante la realización de la tesis doctoral ${ }^{2}$.

Palabras clave: menores migrantes, educación, protección, infancia, inclusión social.

\section{Abstract}

Throughout this article, an approach to the reality of youngtsers will be carried out who, in their day, were received in the System of Protection of the Autonomous Community of Andalusia as migrant minors.

Keywords: migrant minors, education, protection, childhood, social inclusión.

\footnotetext{
${ }^{1}$ Recibido: 10/07/2018 Evaluado: 22/07/2018 Aceptado: 25/08/2018

${ }^{2}$ La reflexión forma parte de la tesis de doctorado (en realización) sobre los menores migrantes en el Programa de Doctorado en Estudios Migratorios de la Universidad de Granada bajo la dirección del profesor F. Javier García Castaño.
} 
This approach will be carried out through the analysis of 42 interviews carried out with youngsters, among which are 38 subjects belonging to the male sex and 4 to the female, stopping in those aspects related to education, training practices and work.

Interviews have been carried out through the different waves of field work carried out during the completion of the doctoral tesis.

La migración internacional no es un fenómeno novedoso, sino que ha estado presente a lo largo de toda la historia de la humanidad (Messina, 2006). Siguiendo a autores como Arango (2007), podemos decir que los desplazamientos han estado condicionados por diversos factores: económicos, políticos, sociales y religiosos, dando lugar a desplazamientos tanto voluntarios como forzosos de la población implicada. A pesar de su existencia a lo largo del tiempo es, en este momento, cuando las migraciones se han convertido en uno de los fenómenos que a nivel nacional, europeo e internacional ocupan un papel relevante en los ámbitos jurídico, social y político, llegándose a hablar incluso de la "Era de la migración" (Castles y Millers, 2004).

Si bien el fenómeno de las migraciones, tradicionalmente, estaba considerado algo como propiamente de adultos, en las últimas décadas se ha identificado la presencia de nuevos actores: los menores migrantes.

España, actualmente, ocupa un puesto clave dentro de las rutas migratorias debiéndose esto, en gran parte, a los avances que se han producido en el transporte, al acceso a las nuevas tecnologías y, también, a una economía de mercado que fomenta las desigualdades (Quiroga y Alonso, 2009). El flujo transnacional constante de mercancías, capital, personas y de información ha provocado la incorporación a la emigración de nuevos grupos sociales, entre los que se encuentran los menores migrantes. Por tanto y, siguiendo a Bravo Rodríguez (2005), el fenómeno migratorio debemos enmarcarlo en el contexto de cambios económicos, sociales, culturales y tecnológicos en las últimas décadas con el avance de la globalización.

La llegada de menores de edad que emprenden el proyecto migratorio en solitario o acompañados de adultos que no ejercen tutela sobre ellos se han consolidado como un nuevo patrón migratorio, que debe ser analizado de manera independiente a la llevada a cabo por los adultos (UNICE, 2009). La presencia de menores migrantes en el Estado Español ya ha cumplido más de 15 años (Quiroga y Alonso, 2009).

Suárez (2006:37) considera que: "los menores extranjeros se constituyen como un nuevo actor migratorio y que, por lo tanto, se puede configurar como una categoría analítica con identidad propia. Este nuevo actor presentaría motivaciones y objetivos propios con una inserción en espacios migratorios más amplios y diferentes". 
Los estudios realizados en ámbitos diversos dentro de España (VVAA, 2004; Calvo de León, 2003; Save The Children, 2005; Capdevilla y Ferrer, 2004; Ramírez y Jiménez, 2005) citados en Goenechea Permisán (2005) y en Hadjab (2017), coinciden al apuntar una serie de características propias de los menores migrantes que nos permiten describir ampliamente este colectivo, entre las que podemos mencionar:

1. La gran mayoría son varones, pudiendo considerarse minoritarios los casos de niñas.

2. En un alto porcentaje proceden de Marruecos (entre el $75 \%$ y el $92 \%$ según los estudios), llamando la atención el hecho de que más de la mitad de los menores migrantes en un estudio realizado en Cataluña procedían de Tánger. No obstante, se apunta a una creciente presencia de menores procedentes de África Subsahariana y, más recientemente, de Europa del Este.

3. La edad de llegada oscila entre los 14 y 17 años, llegando a documentarse casos de menores de 9 años. No obstante, se pone en entredicho la fiabilidad de las pruebas de determinación de la edad.

4. La mayor parte de los menores migrantes vivían con su familia nuclear en su país de origen, coincidiendo en afirmar que no se trata de niños de la calle en sus países.

5. Las circunstancias personales y sociofamiliares no eran buenas, la vivienda tenía poco espacio y disponían de pocos medios para la subsistencia. Estos hechos llevaban a los menores migrantes a abandonar la escuela y a buscar trabajo y, al no encontrarlo, se afianza la idea de emigrar.

6. Los valores y relaciones de los menores migrantes se fundamentan en parámetros culturales diferentes a los nuestros, debiendo esto ser algo a tener en cuenta, en todo momento, en la intervención.

7. Grandes carencias en los ámbitos de escolarización y de formación laboral.

8. Presentan expectativas personales irreales, encontrándose éstas distorsionadas debido a, en parte, los medios de comunicación y a los propios compañeros.

9. Viven una fuerte ruptura de expectativas, debido a la tensión permanente entre la realización de su proyecto migratorio (que incluye responsabilidades de adulto, ser autosuficiente y hasta sustentador de la familia) y la condición de menor migrante (dependiente, en situación de desamparo, sobre el que el Estado tiene competencias de intervención) que les impone la sociedad de acogida.

10. Las motivaciones para emigrar de estos menores coinciden con las de sus compatriotas adultos (un 84\% afirma que emprendió el viaje con la intención de buscar trabajo).

11. Encuentran dificultades para su adaptación e integración, presentando ciertos problemas de convivencia. Además, presentan una grave falta de recursos materiales y familiares para afrontar la situación en la que se encuentran, suponiendo esto un gran riesgo para caer en redes de explotación.

12. Desconocimiento del medio en el que se encuentran y del idioma del país de acogida.

13. Presentan situaciones de desarraigo, soledad, etc.

14. Aparece un nomadismo constante, debido a la gran movilidad que presentan y siendo éste, uno de los principales factores que dificulta su contabilización.

15. La familia no es un elemento activo en la planificación del viaje del menor, aunque en la mayoría de los casos se muestra de acuerdo o se resigna a ello.

16. El viaje migratorio se suele planificar con el grupo de iguales $\mathrm{y}$, frecuentemente, se realiza solo, utilizando para ello, los bajos de camiones, autobuses y pateras. 
Por su parte, Rosa María Bravo Rodríguez, citada en Lázaro González (2007), identifica los siguientes rasgos en el perfil de los menores migrantes:

1. Fundamentalmente son varones.

2. Suelen tener bastantes hermanos.

3. La mayor parte de los menores proceden de Marruecos.

4. Presentan una edad media de 16 años.

5. Su grado de madurez es superior al que le corresponde con su edad cronológica.

6. Mantienen relaciones periódicas con sus familiares de origen.

7. Muestran deseos de mejorar su situación personal y familiar.

8. Su proyecto migratorio se centra en conseguir la documentación y trabajar lo antes posible.

9. Ostentan una gran movilidad geográfica tanto dentro de España, como incluso dentro de Europa, dependiendo de la consecución de sus objetivos.

10. Suelen tener redes de compatriotas que facilitan el acceso.

11. Prefieren el alojamiento en pisos donde puedan tener una mayor autonomía, rechazando el sistema de protección ofrecido.

12. Tienen un bajo nivel de cualificación, a pesar de haber estado escolarizados durante varios años en los países de origen.

13. Suelen tener algún tipo de experiencia laboral.

14. Se decantan por aquellos tipos de actividades formativas que le permitan conseguir un empleo, rechazando la escolarización obligatoria.

15. No se relacionan demasiado con jóvenes autóctonos.

16. Sufren un gran choque entre las expectativas que tenían al venir a España y la realidad a la que deben hacer frente.

17. No contemplan la posibilidad de retorno a sus países de origen.

18. Conocen la legislación y el sistema de protección y hacen uso de él.

Además, Goenechea (2006), Ramírez y Jiménez (2005), Suárez- Navas (2004) y Aparicio Gómez (2009), realizan una clasificación por perfiles de los menores migrantes acogidos en los diversos sistemas de protección del Estado Español, destacando principalmente cuatro perfiles predominantes:

1. La gran mayoría son varones, siendo muy pocos, casi anecdóticos, los casos de niñas o mujeres no acompañadas con edades comprendidas entre los 14 y 18 años.

2. Hasta el año 2006, el perfil social y geográfico central era el de los menores migrantes marroquíes pero, a partir de ese año, comienzan a tener una creciente importancia dentro de este colectivo en España, los menores procedentes de otras partes de África y los procedentes del este de Europa, especialmente de Rumanía. Este cambio en el origen geográfico ha sido fundamental para diversificar y transformar la realidad de los menores migrantes en España y las diferentes líneas de intervención con ellos.

3. La gran mayoría de los menores vivían con sus familias en los países de origen, siendo muy pocos los casos de menores que se podían definir como niños de la calle en sus localidades de procedencia. 
4. Presentan grandes carencias en términos escolares y de formación profesional, bien por no haber estado escolarizados o bien, por haber sufrido procesos de escolarización muy breves por falta de medios económicos.

El acceso a la educación así como la preparación sociolaboral de los menores migrantes para la emancipación y vida autónoma, son aspectos que adquieren una importante relevancia desde el momento de su acogida. Por esta razón, a través del análisis de la información desprendida de las 42 entrevistas llevadas a cabo, nos detendremos en analizar aquella información relativa al paso por el sistema educativo de estos menores migrantes así como su acceso a prácticas formativas e inserción laboral, ejes fundamentales en la inclinación hacia la inclusión o exclusión social.

\section{Paso por el sistema de protección: aspectos educativos, prácticos y laborales}

Antes de pasar a analizar los aspectos educativos, prácticos y laborales del paso de estos jóvenes extutelados por el sistema de protección, resulta necesario, en primer lugar, hacer referencia a una serie de características de la muestra utilizada para este estudio así como a las técnicas de investigación social aplicadas.

En primer lugar, es necesario aclarar que la totalidad de las entrevistas se han realizado a jóvenes extutelados que fueron acogidos en calidad de menores migrantes en centros de protección de las provincias de Almería y Granada.

Las entrevistas en la provincia de Granada se realizaron entre marzo y junio del año 2014 mientras que, en la provincia de Almería, se llevaron a cabo entre enero de 2015 y mayo de 2016.

Para la elección de los sujetos que iban a ser objeto de estudio, se establecieron como criterios las siguientes variables:

- Se eligió como tramo de edad de corte entre 18 y 20 años, existiendo una sola excepción de un joven que tenía la edad de 25 años.

- Nacionalidad. Se buscó a sujetos procedentes de la zona del Magreb y de la zona subsahariana, siendo éstos los que mayor presencia tienen en los centros de protección andaluces.

- Jóvenes con posesión de un buen nivel de comprensión y expresión verbal en castellano.

- Jóvenes migrantes que hubiesen estado bajo la tutela de la Junta de Andalucía y acogidos en los diferentes recursos de protección de menores de esta Comunidad Autónoma hasta alcanzar su mayoría de edad.

En cuanto al perfil de los jóvenes entrevistados, observando la siguiente tabla podremos ver datos relacionados con la edad que ostentaban en el momento de la entrevista, el sexo y el país de origen. 
Tabla 1. Perfil de jóvenes extutelados/as entrevistados/as

\begin{tabular}{|c|c|c|c|}
\hline Entrevista & Sexo & Edad & País de Origen \\
\hline E1 & Masculino & 18 & Marruecos \\
\hline E2 & Masculino & 18 & Argelia \\
\hline E3 & Masculino & 18 & Marruecos \\
\hline E4 & Masculino & 18 & Marruecos \\
\hline E5 & Masculino & 18 & Marruecos \\
\hline E6 & Masculino & 18 & Marruecos \\
\hline E7 & Masculino & 18 & Marruecos \\
\hline E8 & Masculino & 18 & Marruecos \\
\hline E9 & Masculino & 18 & Marruecos \\
\hline E10 & Femenino & 18 & Nigeria \\
\hline E11 & Masculino & 19 & Marruecos \\
\hline E12 & Femenino & 18 & Costa de Marfil \\
\hline E13 & Masculino & 18 & Argelia \\
\hline E14 & Masculino & 19 & Argelia \\
\hline E15 & Masculino & 18 & Marruecos \\
\hline E16 & Masculino & 18 & Marruecos \\
\hline E17 & Masculino & 18 & Argelia \\
\hline E18 & Femenino & 18 & Nigeria \\
\hline E19 & Masculino & 18 & Marruecos \\
\hline E20 & Masculino & 19 & Argelia \\
\hline E21 & Masculino & 19 & Guinea Conakry \\
\hline E22 & Masculino & 18 & Argelia \\
\hline
\end{tabular}




\begin{tabular}{|c|c|c|c|}
\hline E23 & Masculino & 20 & Marruecos \\
\hline E24 & Masculino & 18 & Camerún \\
\hline E25 & Masculino & 18 & Guinea Conakry \\
\hline E26 & Masculino & 18 & Argelia \\
\hline E27 & Masculino & 18 & Argelia \\
\hline E28 & Masculino & 20 & Argelia \\
\hline E29 & Masculino & 18 & Marruecos \\
\hline E30 & Masculino & 18 & Costa de Marfil \\
\hline E31 & Masculino & 18 & Guinea Conakry \\
\hline E32 & Masculino & 18 & Argelia \\
\hline E33 & Masculino & 18 & Argelia \\
\hline E34 & Masculino & 18 & Argelia \\
\hline E35 & Masculino & 18 & Marruecos \\
\hline E36 & Masculino & 18 & Marruecos \\
\hline E37 & Masculino & 18 & Marruecos \\
\hline E38 & Masculino & 20 & Marruecos \\
\hline E39 & Masculino & 20 & Ghana \\
\hline E40 & Masculino & 25 & Marruecos \\
\hline E41 & Femenino & 18 & Marruecos \\
\hline E42 & Masculino & 18 & Marruecos \\
\hline
\end{tabular}

Fuente: elaboración propia.

Por su parte, la técnica de investigación social empleada para la obtención de la siguiente información fue la entrevista en profundidad, considerándola la más adecuada al permitir la emergencia del libre discurso de los jóvenes extutelados/as en torno a sus experiencias vividas a lo largo de sus proyectos migratorios, a su paso por el sistema de protección y sobre cómo se ha producido el tránsito a la mayoría de edad. 
Durante la realización de las entrevistas a estos jóvenes, se pretendió recoger los datos fundamentales respecto a tres bloques diferenciados:

- Bloque 1: "Vida en el país de origen y motivación del viaje". Durante este bloque se trataban temáticas tales como el ámbito familiar, relaciones sociales, situación socioeconómica así como la escolarización y el trabajo.

- Bloque 2: "Paso por el sistema de protección". En este bloque se puso énfasis en el conocimiento de su paso por los centros de protección, por la escuela, dificultades en el proceso de escolarización, su nivel de inserción y de aprendizaje del castellano, la relación con el personal de los centros de protección y el apoyo y seguimiento recibidos, el grado de autonomía alcanzado así como la preparación para el acceso a la mayoría de edad.

- Bloque 3: "Mayoría de edad". Con este bloque se pretendía tener conocimiento acerca de los cambios que se produjeron en la vida de los jóvenes una vez cumplieron la mayoría de edad, si pudieron acceder a uno de los recursos para extutelados y las dificultades encontradas a nivel laboral, asistencial y de documentación.

El orden de los bloques y de las preguntas, responde al enfoque del embudo (kvale, 2008:85), es decir, se comienza la conversación con cuestiones generales, como son las sociodemográficas y, a medida que avanza la entrevista, se va concretando más la temática de la investigación. De igual modo, no todas las preguntas del guión se formularon, ya que al ser una conversación abierta, algunas de las cuestiones se respondían por sí solas sin necesidad de formular la pregunta (Vicente, 2015:201). Y, de la misma forma, ocurrió en caso contrario, es decir, en diversas ocasiones era necesario realizar más preguntas sobre un cierto tema porque emergían nuevas categorías o por lo relevante de la información

Cuadro de texto 1: guión de entrevista en profundidad a jóvenes extutelados/as.

\section{Bloque 1: vida en el país de origen y motivación del viaje:}

¿puedes contarnos algo sobre ti? ¿de dónde eres? ¿qué edad tienes? ¿tienes familia? ¿estudiabas o trabajabas en tu país? ¿cuáles son tus motivaciones para iniciar el viaje? ¿tomaste la decisión solo? ¿viajaste con alguien? ¿cuánto pagaste? ¿cuánto duró? ¿en qué condiciones se realizó el viaje?

\section{Bloque 2: paso por el sistema de protección:}

¿cómo fue tu llegada a España? ¿cómo recuerdas tus primeros días en el centro? ¿cómo era tu relación con el personal que trabajaba allí? ¿te matriculaste en algún curso/formación profesional, etc? ¿te resultó difícil aprender castellano? ¿querías aprenderlo? ¿qué te pareció el instituto? ¿¿tenías horarios de estudio en tu centro? ¿te ayudaban los educadores? ¿qué cambiarías? ¿en cuántos centros de protección has estado? ¿por qué cambiantes de centro? ¿fue difícil para ti el cambio? ¿te has llegado a sentir rechazado? ¿en el centro te ayudaron a tramitar tu documentación? ¿te explicaron bien cómo iba el proceso y qué era necesario para obtener la documentación? ¿te prepararon para la mayoría de edad? ¿pudiste hacer prácticas para encontrar trabajo? ¿qué cambiarías de tu paso por el sistema de protección?

Fuente: elaboración propia. 
A continuación, pasamos a desglosar la información obtenida relativa a los aspectos educativos y laboral de estos jóvenes en su paso por los recursos de protección de menores de la comunidad andaluza.

\subsection{Aspectos educativos.}

Para conocer de cerca el paso de los menores migrantes por el sistema de protección en la Comunidad Autónoma de Andalucía, utilizaremos la información desprendida de las diversas entrevistas llevadas a cabo a jóvenes extutelados/as.

Para ello, en primero lugar, nos vamos a detener en el ámbito escolar-formativo, tratando de analizar cuántos menores fueron matriculados, en qué fueron matriculados y cuántos acabaron dicha formación.

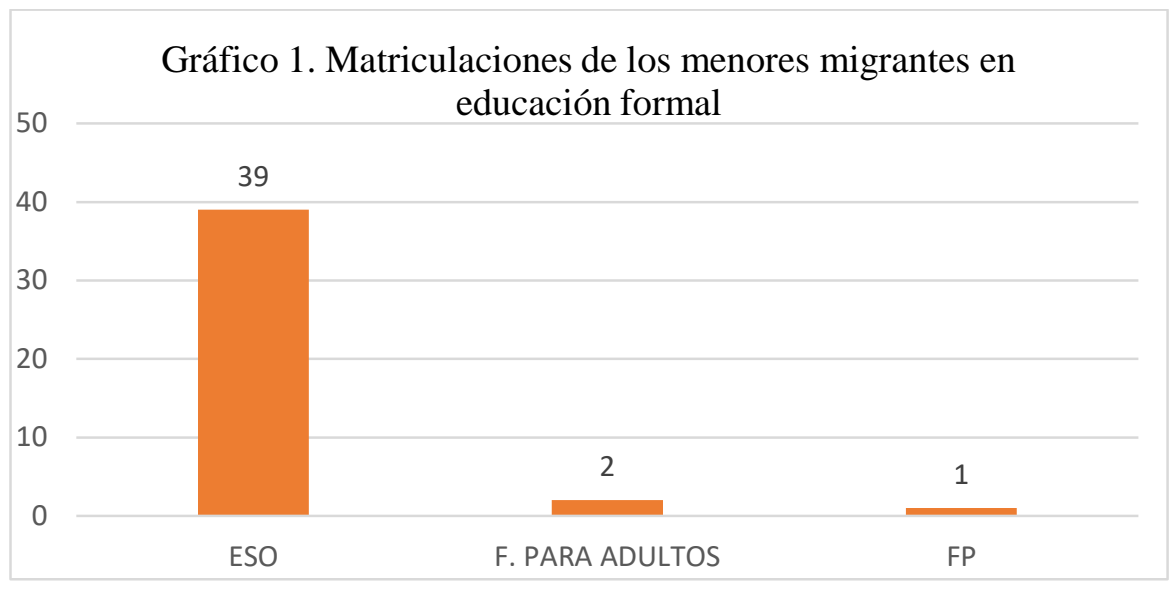

Fuente: elaboración propia a partir de los resultados de la investigación.

En el caso de los 42 jóvenes entrevistados, un total de 39 fueron matriculados en la Educación Secundaria Obligatoria, 2 en Educación para Adultos y 1 de ellos en Formación Profesional.

Cómo puede verse, la mayoría de estos menores fueron matriculados en la Educación Secundaria Obligatoria, a pesar de que la edad de llegada a España sobrepasaba los 16 años. 


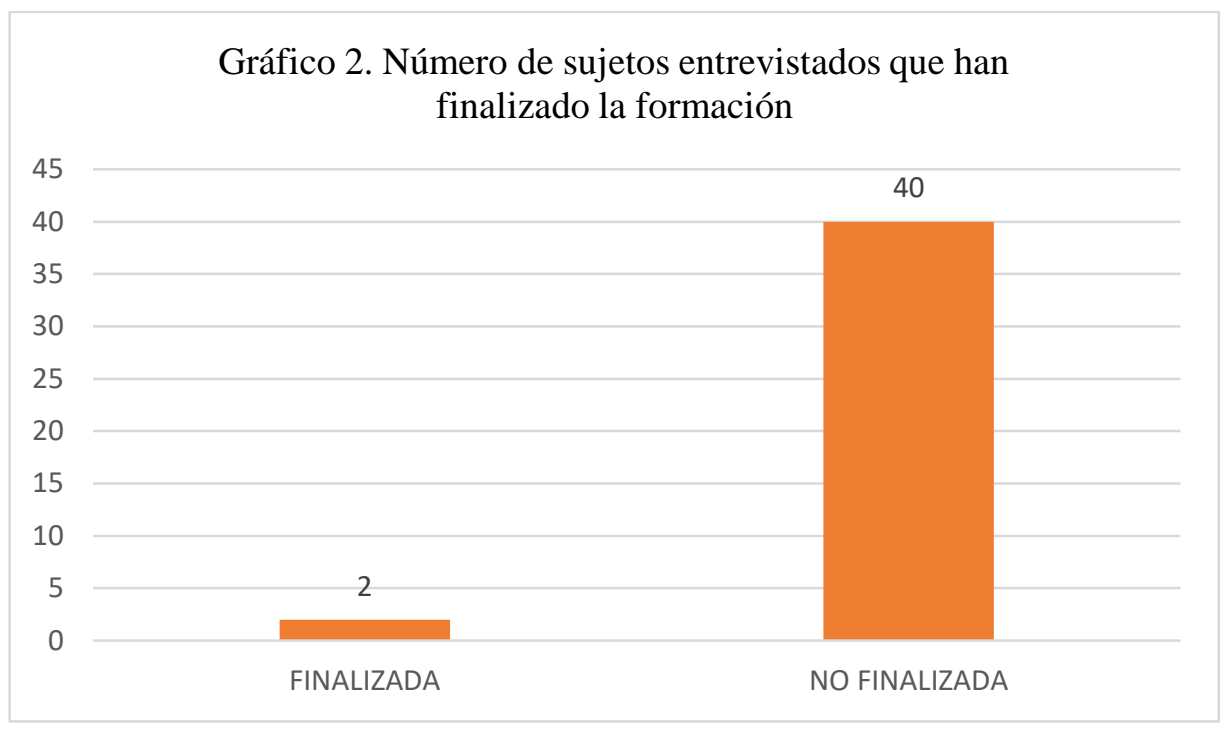

Fuente: elaboración propia a partir de los resultados de la investigación.

Tal y como podemos observar en el gráfico anterior, del total de jóvenes entrevistados, tan solo 2 han terminado la formación en la que se encontraba matriculado siendo, en este caso, la Educación Secundaria Obligatoria.

Otro aspecto a destacar en relación a la formación de estos jóvenes es que, de los 42 entrevistados, todos fueron cambiados de centro de protección al menos en una ocasión, encontrándose ambos en diferentes localidades. Y, un total de 38 jóvenes, fueron cambiados entre 2 y 3 veces de centro, encontrándose en diferentes provincias o localidades los centros de protección a los que debían ser trasladados.

Los numerosos cambios de recursos de protección a los que se enfrentan los menores migrantes obstaculizan, en gran medida, la finalización de todas aquellas actividades formativas iniciadas. Esta cuestión es, verdaderamente, importante tenerla en cuenta ya que la no finalización de las acciones formativas conlleva consecuencias en la inserción laboral así como en la renovación de la documentación de dichos jóvenes, presentando grandes déficits a nivel de aprendizaje (Rodríguez Gómez, 2006: 256).

También resulta de vital importancia hacer mención a que la totalidad de los jóvenes, en el momento de ser matriculados, hubiesen preferido asistir a cursos formativos con la posibilidad de realizar prácticas en empresas o haber sido matriculados en formación profesional y no en la Educación Secundaria Obligatoria, coincidiendo todos ellos que el personal del recurso de protección en ningún momento los entrevistó para saber qué necesidades tenían o cuáles eran sus preferencias e inquietudes hacia el ámbito formativo o laboral.

De las entrevistas realizadas a los diferentes agentes sociales, en relación al ámbito educativo, todos coinciden en las dificultades existentes para la matriculación de los menores migrantes en la Educación Secundaria Obligatoria, obteniendo muchas trabas y negativas por parte de los diferentes centros educativos, basándose en argumentos como la conflictividad, bajo nivel y poca integración de los menores migrantes así como en la posible repercusión social que puede tener a nivel local la asistencia de dichos jóvenes en esos centros educativos. 


\subsection{Aspectos prácticos y laborales.}

En segundo lugar, nos vamos a detener en el ámbito laboral, analizando el número de jóvenes que pudieron realizar prácticas formativas en empresas y en aquellos que, finalmente, consiguieron un contrato de trabajo mientras se encontraban en los recursos de protección.

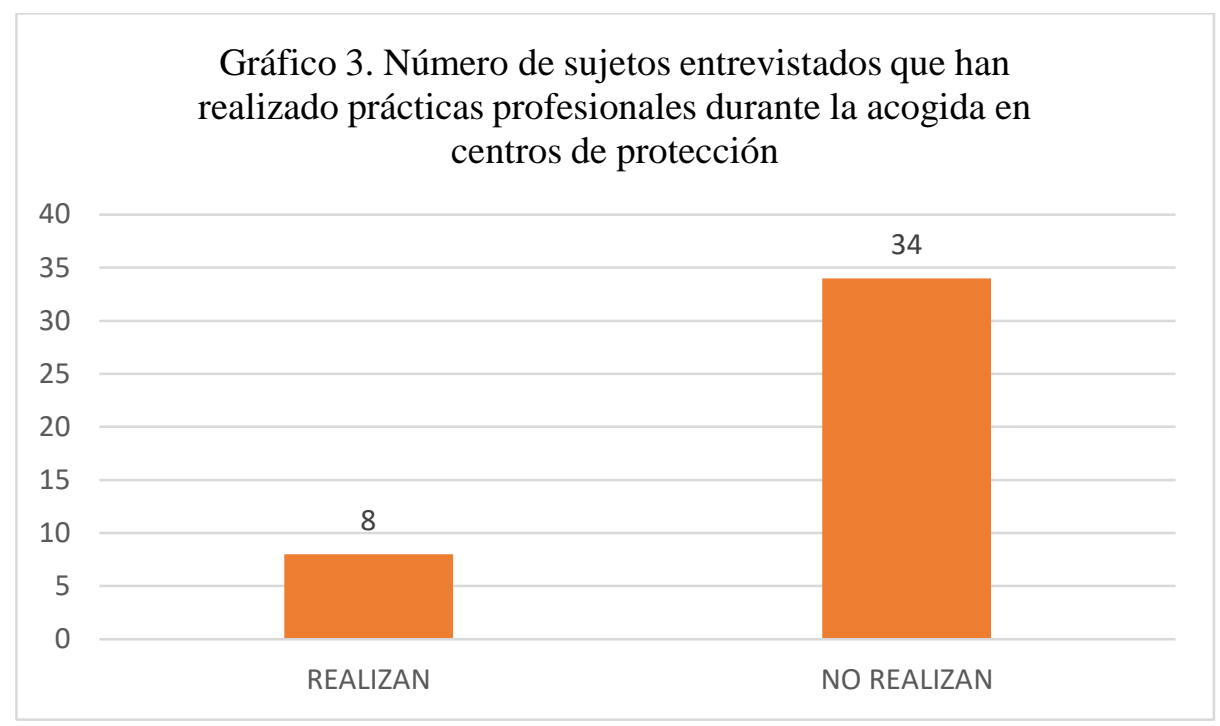

Fuente: elaboración propia a partir de los resultados de la investigación.

En este primer gráfico, podemos observar que de los 42 jóvenes extutelados/as entrevistados, durante su acogida en la red de recursos de protección de menores de la Junta de Andalucía, tan solo accedieron a la posibilidad de realizar prácticas en empresas un total de 8 jóvenes, no siendo ninguno de los mismos del sexo femenino.

En cuanto al ámbito en el que realizaron las prácticas, 6 de ellos las llevaron a cabo en el ámbito agrícola coincidiendo, en su totalidad, que, a pesar de que a nivel formal era un contrato de prácticas, en realidad estaban entre 6 y 8 horas al día trabajando en el campo. Otro de ellos realizó prácticas como mecánico, a través del ciclo de formación profesional que se encontraba realizando. Y, otro de ellos, realizó prácticas en una panadería.

Por último, tal y como podemos comprobar en el gráfico $\mathrm{n}^{\circ} 4$, de los 42 entrevistados tan sólo 4 tuvieron acceso a un contrato laboral de una duración de 6 meses, llevándose a cabo 3 de los mismos en el ámbito agrícola y el restante en una panadería. 


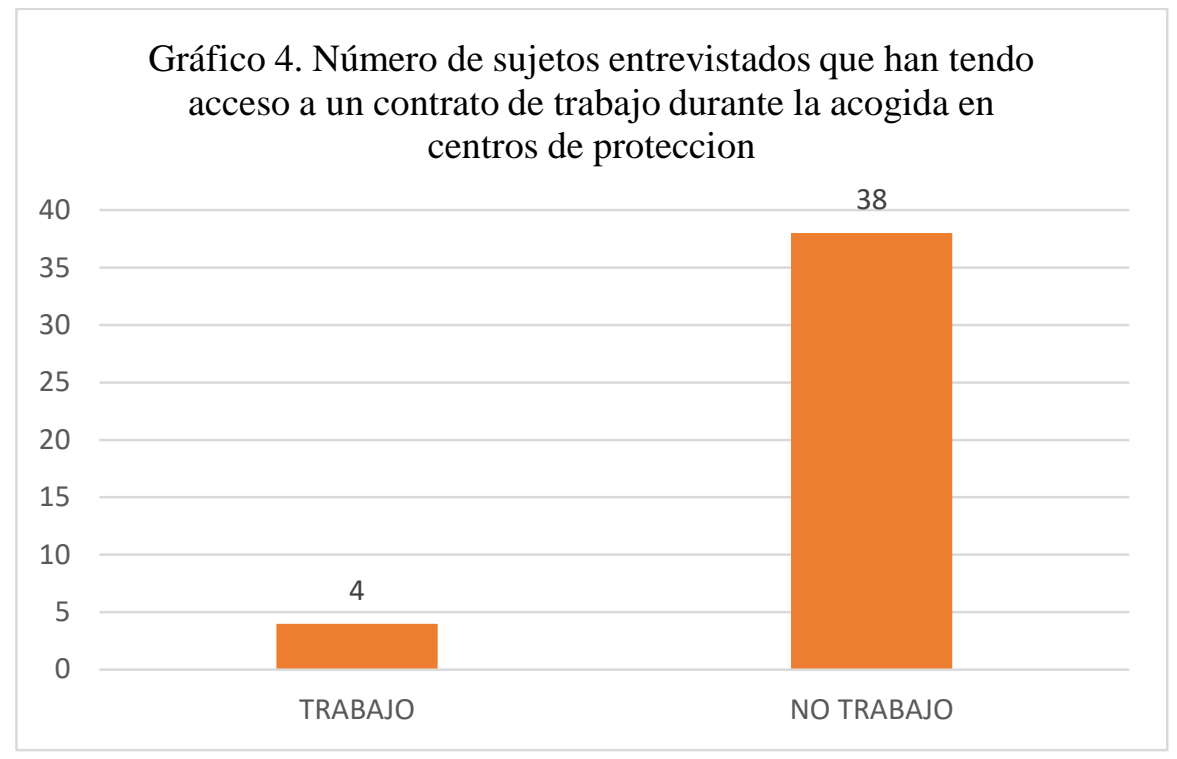

Fuente: elaboración propia a partir de los resultados de la investigación.

Llegados a este punto, es necesario añadir que se ha puesto en funcionamiento un Programa denominado "Programa de Orientación e Inserción Sociolaboral" que se lleva a cabo desde diversos centros de protección y que tiene como objetivo la preparación para la vida independiente así como el desarrollo de programas de formación e inserción laboral de los menores que se encuentran acogidos en Andalucía.

Por su parte, el 16 de julio de 2015, ha sido aprobada en el Congreso de los Diputados la Ley 26/2015, de 28 de julio, de modificación del sistema de protección a la infancia y a la adolescencia. Esta ley contempla en su articulado referencias a la necesidad de que las Entidades Públicas dispongan de programas y recursos destinados al apoyo y orientación de quienes, estando en acogimiento, alcancen la mayoría de edad y queden fuera del sistema de protección (art. 11.4), así como programas de preparación para la vida independiente dirigidos a los jóvenes que estén bajo una medida de protección, particularmente en acogimiento residencial (art.22 bis).

En Andalucía, la Ley 1/1998, de 22 de abril, de los derechos y la atención al menor, en su artículo 37.2 se establece que "al menos, durante el año siguiente a la salida de los menores de un centro de protección, la Administración de la Junta de Andalucía efectuará un seguimiento de aquéllos al objeto de comprobar que su integración sociolaboral sea correcta, aplicando la ayuda técnica necesaria”. Y, en su artículo 19.1.f. que "se potenciará el desarrollo de programas de formación profesional e inserción laboral de los menores sometidos a medidas de protección, con el fin de facilitar su plena autonomía e integración social al llegar a su mayoría de edad”.

En el caso de los jóvenes entrevistados, de las 42 entrevistas llevadas a cabo un total de 12 jóvenes habían estado acogidos, durante sus últimos años en el sistema de protección de menores andaluz, en centros residenciales que desarrollaban el Programa de Orientación e Inserción Sociolaboral.

Sin embargo, los 12 coinciden en que la preparación y formación que han obtenido por medio de estos programas tan sólo se ha basado en el desarrollo de diversos talleres de habilidades sociales, habilidades domésticas y la asistencia a diversos cursos del Programa FormaJoven, que nada tenían que ver con la adquisición de habilidades laborales o formativas para enfrentarse a la vida autónoma 
una vez abandonaran los recursos de protección. Además, también coinciden en afirmar que han echado en falta, a lo largo de todo su acogimiento, explicaciones acerca de cómo debían llevar a cabo los trámites relativos a su documentación, qué documentación era necesaria para poder solicitar el pasaporte o permiso de residencia y que, además, han llegado a desconocer incluso cuál era el estado de tramitación de su documentación así como no tener acceso a información relativa a las fechas en las que debían acudir a los respectivos Consulados o a la Oficina de Extranjería para la gestión de su documentación.

Otro aspecto a destacar en relación a los centros que desarrollan los Programas de Orientación e Inserción Sociolaboral es su lejanía, es decir, se encuentran ubicados en localidades donde las oportunidades laborales y formativas para los jóvenes se reducen considerablemente, no existiendo la posibilidad de acudir, diariamente, a la ciudad más cercana para la búsqueda de dichas oportunidades, ostentando argumentos como: poca financiación, lejanía y escasez de medios de transporte que comunique dichas localidades con la capital de provincia.

Cuadro de texto 2: fragmento de entrevistas realizadas a jóvenes extutelados.

"Los centros deben estar en sitios menos apartados y, seguramente, para que no hubiera problemas con los vecinos, deberían ser más pequeños".

"Es cierto que nosotros no somos igual que los españoles que se encuentran en los centros, pero eso no quiere decir que no deban procurar que tengamos acceso a una preparación suficiente para cuando debamos abandonar los centros ya que, muchas veces, parece que nos abren la puerta del centro y nos dejan tirados, da la sensación de que no les importamos y que nuestra formación tampoco es importante, porque no hablamos bien español".

Fuente: elaboración propia en base a los datos de la investigación.

Por su parte, los agentes sociales entrevistados, reiteran que los jóvenes que se encuentran acogidos en aquellos centros que desarrollan los Programas de Orientación e Inserción Sociolaboral, en muchas ocasiones, no responden al perfil que se espera que se encuentre acogido en dichos centros, atendiendo al nivel de idioma, documentación e intereses de los jóvenes.

\section{Discusión y conclusiones}

Cada vez más, son más los menores migrantes que son acogidos en Andalucía con edades cercanas a la mayoría de edad. Por tanto, estamos asistiendo, sobre todo, en los últimos años, a un cambio en los perfiles de acogida así como en las problemáticas a las que, desde los recursos de protección, se ha de hacer frente. Elementos como el acceso a los diversos recursos formativos, la preparación para el empleo o la inserción sociolaboral de los menores migrantes, la preparación para la emancipación y la vida autónoma, la regularización de su situación administrativa, son los ámbitos que adquieren una mayor importancia para ser trabajados y, en la mayor parte de los casos, no existe tiempo material suficiente para preparar al joven para la vida adulta, la tramitación de su documentación y la adquisición de hábitos laborales y formativos suficientes para su inserción sociolaboral.

Un dato importante a tener en cuenta es la formación obtenida o finalizada por parte de los jóvenes entrevistados. La mayor parte de ellos fueron matriculados en la Educación Secundaria Obligatoria que, atendiendo a hechos como su nivel de castellano, sus preferencias, los diferentes cambios de 
centros, así como su edad de ingreso en el sistema de protección de menores andaluz, han dificultado su finalización. Resulta de vital importancia, atendiendo a la edad del menor, que el proceso formativo de los menores vaya acorde a sus intereses así como a las reales oportunidades existentes para su finalización u obtención. En cualquier caso, matricular a un joven de 16 o 17 años en la ESO, a sabiendas de que alcanzará la mayoría de edad y no habrá podido obtener dicho título, nos hace plantearnos la necesidad de valorar la posibilidad de acercarlos a otro tipo de formación académica que les facilite la inserción sociolaboral una vez cumplan la mayoría de edad.

En este caso, hemos visto cómo la falta de un programa o itinerario educativo elaborado de forma conjunta con los menores, los numerosos cambios de centro, y los cambios de perfil en cuanto a la edad con la que se acoge a estos menores, afectan de forma considerable en los logros a nivel formativo de los menores migrantes. Resulta necesario reducir al máximo el número de cambios de centro en los recursos residenciales puesto que este hecho repercute, considerablemente, en la posibilidad de finalizar los diferentes cursos académicos o formativos, así como en la estabilidad de los menores.

Los centros residenciales que desarrollan programas de orientación e inserción laboral están destinados a la acogida de menores con edades comprendidas entre los 16 y 18 años, puesto que son éstos los que se encuentran en edad laboral. Sin embargo, no son muchos los centros residenciales que desarrollan este tipo de programa y, además, en ocasiones, atendiendo a las dificultades sobrevenidas de acogida, llegan a funcionar como programas de acogida inmediata.

No obstante, atendiendo al número reducido de centros residenciales con dicho programa, se hace palpable la necesidad de que el resto de recursos residenciales básicos, desarrollen actividades destinadas a la formación e inserción sociolaboral de los jóvenes que se encuentren acogidos en sus instalaciones una vez cumplan los 16 años hasta su mayoría de edad, con el objetivo de avanzar en su autonomía así como preparación para la vida adulta.

Otro aspecto a mejorar en relación a los centros residenciales con programas de orientación e inserción sociolaboral es su ubicación, puesto que la mayor parte de ellos se encuentran alejados de las capitales de provincia, algo que, sin duda, dificulta la búsqueda de empleo entre los menores acogidos así como el acceso a los diferentes cursos de formación laboral.

Siguiendo a Orteu (2008), cumpliendo con las normativas vigentes y con la intención de romper el círculo de la pobreza, marginación social y exclusión a la que se pueden ver abocados estos jóvenes, es importante desarrollar programas o proyectos cuyos objetivos sean: ayudar en los procesos de transición a la vida adulta; ofrecer la oportunidad de vincularse con el mundo del trabajo a los jóvenes extutelados, a través de la adquisición de competencias de empleabilidad y el aprendizaje de conocimientos de carácter pre-laboral.

\section{Referencias}

Arango, J. (2003). Las explicaciones teóricas de las migraciones: Luz y sombra. Migración y Desarrollo, 1,31 .

Boletín Oficial del Estado (1998). Ley 1/1998, de 20 de abril, de los Derechos y la Atención al Menor. Disponible en http://cort.as/-A4Kq

Boletín Oficial del Estado (2015). Ley 26/2015, de 28 de julio, de modificación del sistema de protección a la infancia y a la adolescencia, Pub. L. No. 180.

$\mathrm{N}^{\mathrm{0}} 7,2019$. Página 68 
Boudiaf, H. H. (2017). Las nuevas generaciones de personas menores migrantes (Tesis Doctoral) Universidad de Granada.

Bravo Rodríguez, R. M. (2005). La situación de menores no acompañados en España. In Conferencia Regional sobre las Migraciones de los Menores No Acompañados: actuar de acuerdo con el Interés Superior del Menor. Ministerio de Trabajo y Asuntos Sociales, Torremolinos, Málaga, España.

Calvo de León, R., \& Arroyo Alonso, L. J. (2003). Actuación educativa con menores extranjeros no acompañados en la Residencia Gregorio Santiago de Burgos. Luque, PA; Amador, LV y Malagón, JL (2003). Educación Social e Inmigración. Sevilla: Sociedad Ibérica de Pedagogía Social y Diputación de Sevilla, 227-236.

Capdevila, M. C., \& Puig, M. F. (2004). Estudio sobre los menores extranjeros que llegan solos a Cataluña. Migraciones. Publicación del Instituto Universitario de Estudios sobre Migraciones, (16), 121-156.

Castles, S., Miller, M. J., \& Quiroz, L. R. M. (2004). La era de la migración: movimientos internacionales de población en el mundo moderno (No. 304.82 C3). México: Universidad Autónoma de Zacatecas.

Goenechea Permisán, C. (2002). La investigación sobre menores extranjeros en España: necesidades detectadas en su escolarización. En La inmigración en España: contextos y alternativas (pp. 479-487). Laboratorio de Estudios Interculturales.

Kvale, Steinar (2008). Las entrevistas en investigación cualitativa. Madrid: Ediciones Morata.

Lázaro González, I. (2007). Menores extranjeros no acompañados. La situación en España. Prolegómenos. Derechos y Valores, 10(19).

Messina, A. M., \& Lahav, G. (Eds.). (2006). The migration reader: exploring politics and policies. Boulder, CO: Lynne Rienner.

Navaz, L. S., \& Social, C. D. A. (2006). Un nuevo actor migratorio: jóvenes, rutas y ritos juveniles transnacionales. En Francisco Checa Olmos; Anjeles, Arjona y Juan Carlos Checa Olmos, Menores tras la frontera: otra inmigración que aguarda. Barcelona: Icaria, 17-50.

Orteu, X., Verdeguer, P., \& Venceslao, M. (2008). L'acompanyament acadèmico-professional amb adolescents: un espai d'intersecció on construir una proposta educativa. Interrogant, (8), 4649.

Quiroga, V., \& Alonso, A. (2009). Sueños de bolsillo. Disponible en http://cort.as/-A4Sh

Ramírez Fernández, A., \& Jiménez Álvarez, M. (2005). Las otras migraciones: la emigración de menores marroquíes no acompañados a España. Madrid: Ediciones Akal.

Rodríguez Gómez, D. (2006). Modelos para la creación y gestión del conocimiento: una aproximación teórica. Educar, 37.

Rojo. L. (2009). Estudio sobre la migración internacional de los menores extranjeros no acompañados subsaharianos hacia las islas canarias. Fundación Nuevo Sol: Murcia. Disponible en http://cort.as/-A4Su 
Suarez Navaz, L. (2004). Ninos entre fronteras: migracion de menores no acompanados en el mediterraneo occidental, 35-48.

UNICEF. (2009). Ni ilegales ni invisibles. Realidad jurídica y social de los Menores Extranjeros en España. Disponible en http://cort.as/-A4L7

Vicente, Trinidad (2015). Mujeres inmigrantes: proyectos y realidades del proceso migratorio. Un análisis de la inmigración femenina marroquí, ecuatoriana y colombiana en España. Bilbao: Universidad de Deusto.

Vila-Belda, J. A. (2007). Las migraciones internacionales en un mundo globalizado. En Inmigración en Canarias: contexto, tendencias y retos (pp. 11-22). Fundación Pedro García Cabrera.

VVAA. (2004). Conclusiones del Seminario Europeo "Menores migrantes no acompañados en Europa”. Barcelona, del 12 al 14 de mayo (http://www.peretarres.org). 